\title{
ON MAGNETIC FIELDS IN ROTATING NUCLEAR MATTER CORES OF STELLAR DIMENSIONS
}

\author{
KUANTAY BOSHKAYEV, MICHAEL ROTONDO and REMO RUFFINI \\ Dipartimento di Fisica, Università di Roma Sapienza, \\ Piazzale Aldo Moro 5, I-00185 Roma, Italy, \\ ICRANet, Piazzale della Repubblica 10, I-65122 Pescara, Italy \\ kuantay@icra.it,michael.rotondo@icra.it andruffini@icra.it
}

\begin{abstract}
We consider a degenerate globally neutral system of stellar dimensions consisting of $N_{n}$ neutrons, $N_{p}$ protons and $N_{e}$ electrons in beta equilibrium. Such a system at nuclear density having mass numbers $A \approx 10^{57}$ can exhibit a charge distribution different from zero. We present the analysis in the framework of classical electrodynamics to investigate the magnetic field induced by this charge distribution when the system is allowed to rotate as a whole rigid body with constant angular velocity around the axis of symmetry.
\end{abstract}

Keywords: Thomas-Fermi model; Neutron star core; Magnetic field.

PACS number: 31.15.bt, 71.10.Ca, 26.60.Dd

\section{Introduction}

Neutron stars are mainly detected as pulsars, whose regular pulsations in the radio, $\mathrm{X}$-ray, and optical bands are produced by constant, ordered magnetic fields that are the strongest known in the Universe. However the origin of the magnetic field in the neutron stars is not fully understood, so far. Nevertheless in the literature one may find various hypotheses explaining the formation of the magnetic field $\mathrm{d}^{1,2,3,4,5,6,7}$. The simplest hypothesis to explain the presence of the strong fields observed in neutron stars is the conservation of the magnetic flux already present in the progenitor stars during the gravitational collapse. This idea is based on the assumption that all stars at all stages of their evolution have some magnetic field, due to electronic currents circulating in their interiors. Thus this argument led to the prediction of the fields $B \approx 10^{12} G$ in neutron stars a few years before the discovery of pulsars ${ }^{1,2}$. However, there is no detailed physical picture of such a flux conserving collapse. Thompson and Duncan ${ }^{8}$ put forward the hypothesis that newborn neutron stars are likely to combine vigorous convection and differential rotation making a dynamo process operate in them. They predicted fields up to $10^{15}-10^{16} G$ in neutron stars with few millisecond initial periods, and suggested that such fields could explain much of the phenomenology associated with Soft Gamma Repeaters and Anomalous X-ray Pulsars $^{9,10}$. 
Probably, these processes are not mutually exclusive. A strong field might be present in the collapsing star, but later be deformed and perhaps amplified by some combination of convection, differential rotation, and magnetic instabilities ${ }^{11,12}$. The relative importance of these ingredients depends on the initial field strength and rotation rate of the star. For both mechanisms, the field and its supporting currents are not likely to be confined to the solid crust of the star, but distributed inmost of the stellar interior, which is mostly a fluid mixture of neutrons, protons, electrons, and other, more exotic particles.

Unlike aforementioned hypotheses which are based on the assumptions that all stars are magnetized or charged with some net charge different from zero, we explore the system recently considered by Ruffini et. al. ${ }^{13}$. According to that work the system consisting of degenerate neutrons, protons and electrons in beta equilibrium is globally neutral and expected to be kept at nuclear density by self gravity. In what follows these systems are termed as Nuclear Matter Cores of Stellar Dimensions. Despite the global neutrality the charge distribution turned out to be different from zero inside and outside (near the surface) the star. The magnitude of the net charge inside and outside the core is equal, but the sign is opposite. Such an effect takes place as a consequence of the beta equilibrium, the penetration of electrons into the core, hence the screening of the core charge and global charge neutrality. As a result of this effect, one may show the presence of an electric field close to the critical value $E_{c}=m_{e}^{2} c^{3} / e \hbar$ near the surface of the massive cores, although localized in a very narrow shell. Thus in this case the magnetic field of the neutron star may be generated only if it spins like pulsars, even though the progenitor star has not been magnetized or electrically charged.

\section{The Relativistic Thomas-Fermi equation}

The Thomas-Fermi equation is the exact theory for atoms, molecules and solids as $N_{p} \rightarrow \infty^{14}$. The relativistic Thomas-Fermi theory developed for the study of atoms for heavy nuclei with $N_{p}=10^{615,16,17}$ gives important basic new information on the study of nuclear matter in bulk in the limit of $A=\left(m_{\text {Planck }} / m_{n}\right)^{3}$ nucleons of mass $m_{n}$ and on its electrodynamic properties. The analysis of nuclear matter bulk in neutron stars composed of degenerate gas of neutrons, protons and electrons, has traditionally been approached by implementing microscopically the charge neutrality condition by requiring the electron density $n_{e}(r)$ to coincide with the proton density $n_{p}(r)$,

$$
n_{e}(r)=n_{p}(r)
$$

It is clear however that especially when conditions close to the gravitational collapse occur, there is an ultra-relativistic component of degenerate electrons whose confinement requires the existence of very strong electromagnetic fields, in order to guarantee the overall charge neutrality of the neutron star. Under these conditions equation (1) will be necessarily violated. 


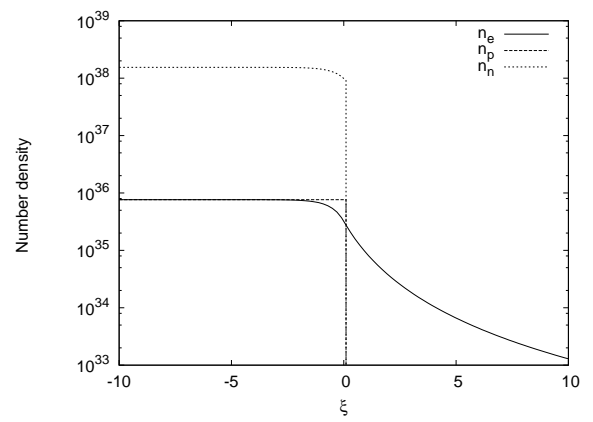

Fig. 1. The neutron number density $n_{n}$, the proton number density $n_{p}$ and the electron number density $n_{e}$, expressed in $\mathrm{cm}^{-3}$, are plotted as functions of the dimensionless radial coordinate $\xi$. It is clear that near the surface of the core is located a transition layer of width $\approx \hbar /\left(\sqrt{\alpha} m_{\pi} c\right)$ with an uncompensated charge which generates an overcritical electric field (see Fig. 4).

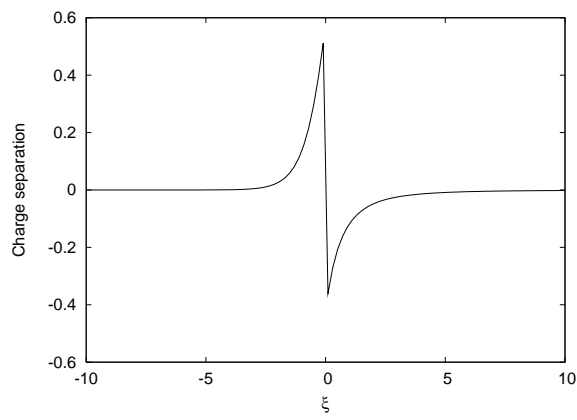

Fig. 2. The normalized charge separation $\left(n_{p}-n_{e}\right) / n_{p}$ is plotted as function of the dimensionless radial coordinate $\xi$. The maximum charge separation happens near the surface of the core where a transition layer with an uncompensated charge is located (see Fig. 1).

Using the relativistic Thomas-Fermi equation, Ferreirinho et al. ${ }^{16}$, Ruffini and Stella $^{17}$ have analyzed the electron densities around an extended nucleus in a neutral atom all the way up to $N_{p}=6000$. They have shown the effect of penetration of the electron orbital well inside the nucleus, leading to a screening of the nuclei positive charge and to the concept of an "effective" nuclear charge distribution.

In the work of Ruffini et. al. ${ }^{13}$ and Popov et. al. ${ }^{18}$ the relativistic ThomasFermi equation has been used to extrapolate the treatment of super heavy nuclei to the case of nuclear matter cores of stellar dimensions. These cores represent the inner part of neutron stars and are characterized by an atomic number of order of $A=\left(m_{\text {Planck }} / m_{n}\right)^{3} \approx 10^{57}$, composed of degenerate neutrons, protons and electrons in beta equilibrium and expected to be kept at nuclear density by self gravity. It has been shown that near the surface of the massive cores it is possible to have an electric field close to the critical value $E_{c}$, although localized in a very narrow shell of the order of the $\lambda_{e}$ electron Compton wavelength. Now let us review the main assumptions and results of those works. According to ${ }^{13}$ and ${ }^{18}$ the protons are distributed at constant density $n_{p}$ within a radius

$$
R_{c}=\Delta \frac{\hbar}{m_{\pi} c} N_{p}^{1 / 3}
$$

where $\Delta$ is a parameter such that $\Delta \approx 1(\Delta<1)$ corresponds to nuclear (supranuclear) densities when applied to ordinary nuclei. The overall Coulomb potential satisfies the Poisson equation

$$
\nabla^{2} V(r)=-4 \pi e\left[n_{p}(r)-n_{e}(r)\right]
$$


with the boundary conditions $V(\infty)=0$ (due to the global charge neutrality of the system) and finiteness of $V(0)$. The density $n_{e}(r)$ of the electrons of charge $-e$ is determined by the Fermi energy condition on their Fermi momentum $P_{e}^{F}$; we assume here

$$
E_{e}^{F}=\left[\left(P_{e}^{F} c\right)^{2}+m_{e}^{2} c^{4}\right]^{1 / 2}-m_{e} c^{2}-e V(r)=0
$$

which leads to

$$
n_{e}(r)=\frac{\left(P_{e}^{F}\right)^{3}}{3 \pi^{2} \hbar^{3}}=\frac{1}{3 \pi^{2} \hbar^{3} c^{3}}\left[e^{2} V^{2}(r)+2 m_{e} c^{2} e V(r)\right]^{3 / 2} .
$$

By introducing the dimensionless quantities $x=r /\left[\hbar / m_{\pi} c\right], x_{c}=R_{c} /\left[\hbar / m_{\pi} c\right]$ and $\chi / r=e V(r) / c \hbar$, the relativistic Thomas-Fermi equation takes the form

$$
\frac{1}{3 x} \frac{d^{2} \chi(x)}{d x^{2}}=-\frac{\alpha}{\Delta^{3}} H\left(x_{c}-x\right)+\frac{4 \alpha}{9 \pi}\left[\frac{\chi^{2}(x)}{x^{2}}+2 \frac{m_{e}}{m_{\pi}} \frac{\chi}{x}\right]^{3 / 2},
$$

where $\alpha=e^{2} /(\hbar c)$ is the fine structure constant, $H\left(x_{c}-x\right)$ is the Heaviside step function and the boundary conditions for $\chi(x)$ are $\chi(0)=0, \chi(\infty)=0$. The neutron density $n_{n}(r)$ is determined by the Fermi energy condition on their Fermi momentum $P_{n}^{F}$ imposed by beta decay equilibrium

$$
E_{n}^{F}=\left[\left(P_{n}^{F} c\right)^{2}+m_{n}^{2} c^{4}\right]^{1 / 2}-m_{n} c^{2}=\left[\left(P_{p}^{F} c\right)^{2}+m_{p}^{2} c^{4}\right]^{1 / 2}-m_{p} c^{2}+e V(r),
$$

which in turn is related to the proton and electron densities by Eqs. (3), (5) and (6).

\section{The ultra-relativistic analytic solutions}

In the ultrarelativistic limit with the planar approximation the relativistic ThomasFermi equation admits an analytic solution. Introducing the new function $\phi$ defined by $\phi=4^{1 / 3}(9 \pi)^{-1 / 3} \Delta \chi / x$ and the new variables $\hat{x}=(12 / \pi)^{1 / 6} \sqrt{\alpha} \Delta^{-1} x, \xi=\hat{x}-\hat{x}_{c}$, where $\hat{x}_{c}=(12 / \pi)^{1 / 6} \sqrt{\alpha} \Delta^{-1} x_{c}$, then Eq. (6) becomes

$$
\frac{d^{2} \hat{\phi}(\xi)}{d \xi^{2}}=-H(-\xi)+\hat{\phi}(\xi)^{3}
$$

where $\hat{\phi}(\xi)=\phi\left(\xi+\hat{x}_{c}\right)$. The boundary conditions on $\hat{\phi}$ are: $\hat{\phi}(\xi) \rightarrow 1$ as $\xi \rightarrow-\hat{x}_{c} \ll 0$ (at the nuclear matter core center) and $\hat{\phi}(\xi) \rightarrow 0$ as $\xi \rightarrow \infty$. The function $\hat{\phi}$ and its first derivative $\hat{\phi}^{\prime}$ must be continuous at the surface $\xi=0$ of the nuclear matter core of stellar dimensions. Hence equation (8) admits an exact solution

$$
\hat{\phi}(\xi)= \begin{cases}1-3\left[1+2^{-1 / 2} \sinh (a-\sqrt{3} \xi)\right]^{-1}, & \xi<0, \\ \frac{\sqrt{2}}{(\xi+b)}, & \xi>0,\end{cases}
$$

where the integration constants $a$ and $b$ have the values $a=\operatorname{arccosh}(9 \sqrt{3}) \approx 3.439$, $b=(4 / 3) \sqrt{2} \approx 1.886$. Next we evaluate the Coulomb potential and electric field 


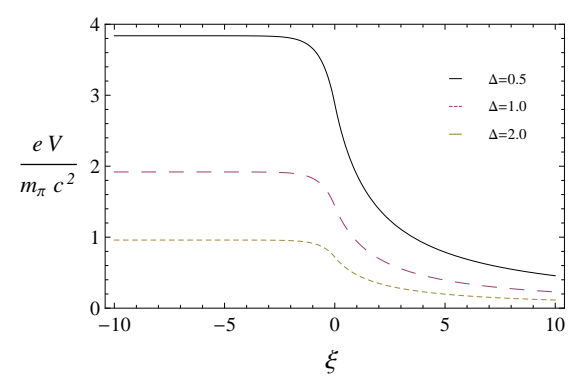

Fig. 3. The proton Coulomb potential energy $\mathrm{eV}$, in units of pion mass $m_{\pi}$ is plotted as a function of the radial coordinate $\xi=\hat{x}-\hat{x}_{c}$, for selected values of the density parameter $\Delta$.

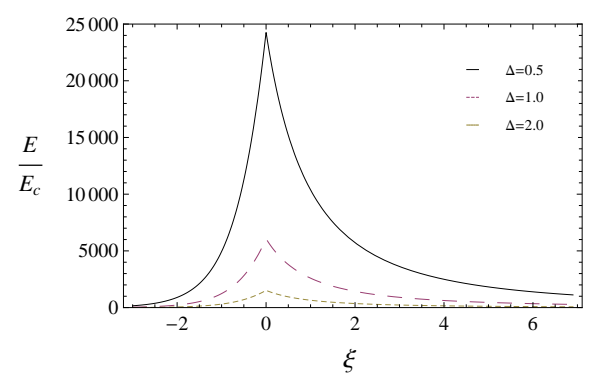

Fig. 4. The electric field is plotted in units of the critical field $E_{c}$ as a function of the radial coordinate $\xi$, showing a sharp peak at the core radius, for selected values of $\Delta$.

functions

$$
V(\xi)=\left(\frac{9 \pi}{4}\right)^{1 / 3} \frac{m_{\pi} c^{2}}{\Delta e} \hat{\phi}(\xi), \quad E(\xi)=-\left(\frac{3^{5} \pi}{4}\right)^{1 / 6} \frac{\sqrt{\alpha}}{\Delta^{2}} \frac{m_{\pi}^{2} c^{3}}{e \hbar} \hat{\phi}^{\prime}(\xi) .
$$

Details are given in Figs. 3 and 4 .

\section{Rotating Nuclear Matter Cores of Stellar Dimensions in Classical Electrodynamics}

In section 2 and 3 we have seen that in the massive nuclear density cores the electric charge distribution is different from zero, although it is globally neutral (for details see Table 1). In this section we investigate the case when this charge distribution is allowed to rotate with the constant angular velocity $\vec{\omega}=\{0,0, \omega\}$ around the axis of symmetry. Thus the magnetic field of the resultant current density is calculated in terms of the charge distribution. Consider a charge distribution moving in a such way that at every point in space the charge density and the current density remain constant. In this case the magnetic field is defined by

$$
\vec{B}(\vec{r})=\vec{\nabla} \times \vec{A}(\vec{r}), \quad \vec{A}(\vec{r})=\frac{\vec{\omega}}{c^{2}} \times \vec{F}(\vec{r}), \quad \vec{F}(\vec{r})=\frac{1}{4 \pi \varepsilon_{0}} \int \frac{\overrightarrow{r^{\prime}} \rho\left(\overrightarrow{r^{\prime}}\right) d^{3} \overrightarrow{r^{\prime}}}{\left|\vec{r}-\overrightarrow{r^{\prime}}\right|}
$$

where $\vec{A}$ is the vector potential of the magnetic field, $\vec{F}(\vec{r})$ is the "superpotential" in general form and $\varepsilon_{0}$ is the electric constant. In the case of spherical symmetry, $\vec{F}(\vec{r})$ may be taken as radial (see Marsh ${ }^{19}$ ). Writing $\vec{F}(\vec{r})=\vec{e}_{r} F(r)$, where $\vec{e}_{r}$ is the unit radial vector, one has

$$
F(r)=\frac{1}{r^{2}} \int_{0}^{r} r^{\prime 2} \frac{d}{d r^{\prime}}\left[r^{\prime} V\left(r^{\prime}\right)\right] d r^{\prime}
$$

This expression allows to calculate the magnetic field induced due to rotation of any spherically symmetric distribution of charge in terms of its electrostatic Coulomb potential. Note that in fact due to rotation the shape of the neutron star must 


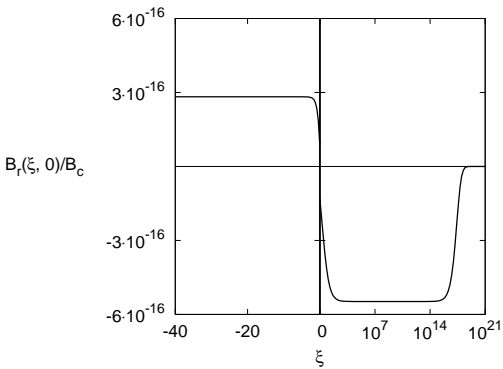

Fig. 5. The radial component of the magnetic field $B_{r}$ is plotted as a function of the radial coordinate $\xi$ in units of the critical field $B_{c}=$ $m_{e}^{2} c^{3} / e \hbar \approx 4.5 \times 10^{13} G$. Here the period is taken to be $P=10 \mathrm{~ms}, \theta=0, \Delta=1$ and the radius of the core $R_{c}=10 \mathrm{~km}$. Note that $B_{r}$ is considered at the poles of star, where it has maximum value. Outside the star $B_{r}$ has very small negative value and it tends to zero.

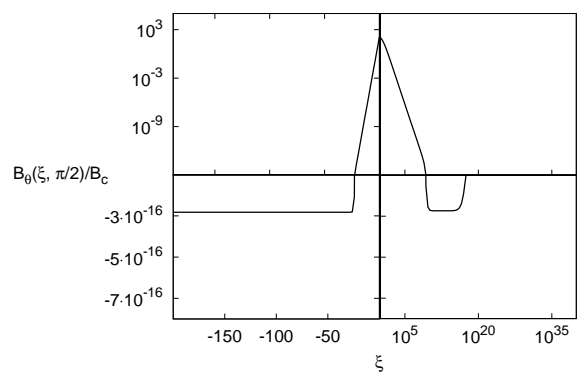

Fig. 6. The angular component of the magnetic field $B_{\theta}$ is plotted in units of the $B_{c}$. Here $P=10 \mathrm{~ms}, \theta=\pi / 2, \Delta=1$ and $R_{c}=10 \mathrm{~km}$. Note that $B_{\theta}$ is considered at the equator, where it has maximum value. Inside the star it has very small constant negative value. Outside the star first it becomes negative (the value is very small) then it tends to zero.

deviate from spherical symmetry. Since we are interested in the estimation of the order of the magnetic field the distortions to the shape of the star can be neglected for simplicity. Thus the magnetic field is defined by

$$
\vec{B}(\vec{r})=B_{r} \vec{e}_{r}+B_{\theta} \vec{e}_{\theta}
$$

where

$$
B_{r}=\frac{2 \omega}{c^{2}} \frac{F}{r} \cos \theta, \quad B_{\theta}=-\frac{2 \omega}{c^{2}}\left[\frac{F}{r}+\frac{r}{2} \frac{d}{d r}\left(\frac{F}{r}\right)\right] \sin \theta,
$$

$B_{r}$ is the radial component and $B_{\theta}$ is the angular component of the magnetic field, $\theta$ is the angle between $r$ and $z$ axis, and $\vec{e}_{\theta}$ is the unit vector along $\theta$. Consequently the expression for the magnitude (the absolute value) of the magnetic field can be written as

$$
B(r, \theta)=\frac{\omega r}{c^{2}} \sqrt{\left(\frac{2 F}{r^{2}}\right)^{2}+\left\{\frac{4 F}{r^{2}} \frac{d}{d r}\left(\frac{F}{r}\right)+\left[\frac{d}{d r}\left(\frac{F}{r}\right)\right]^{2}\right\} \sin ^{2} \theta} .
$$

Using the relation between $r$ and $\xi$

$$
r=R_{c}+\left(\frac{\pi}{12}\right)^{1 / 6} \frac{\Delta}{\sqrt{\alpha}} \frac{\hbar}{m_{\pi} c} \xi,
$$

one may estimate the value of the magnetic field. Details are given in Figs. 5, 6, 7 and 8 .

Examining the Fig. 5 one can see very small value of $B_{r}$ which almost does not make a significant contribution to the magnitude of the field, except for the poles of 


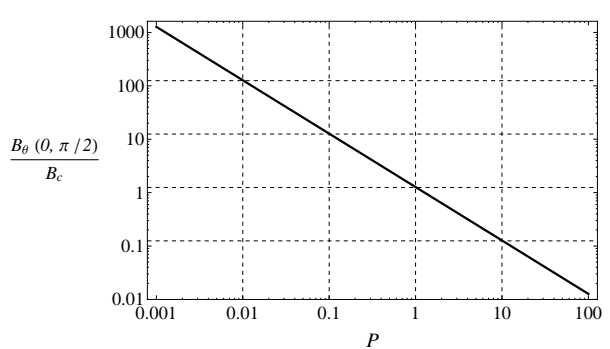

Fig. 7. The magnitude of the magnetic field is plotted as a function of the period of the star $P$ in the units of the critical field $B_{c}$ at the surface of the core $R_{c}=10 \mathrm{~km}$ on the equator in the logarithmic scale.

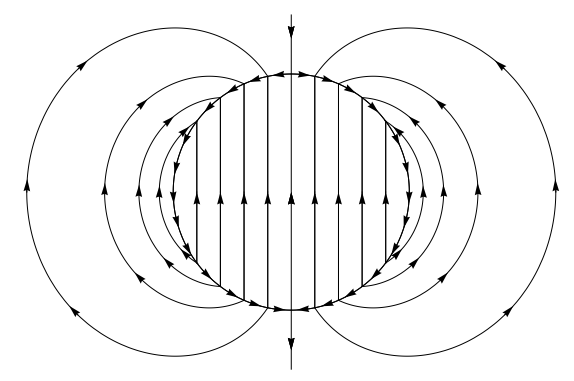

Fig. 8. A schematic illustration of the magnetic lines of forces. Outside the star the magnetic field looks like a dipole field. Extra lines (arrows) along the surface of the star indicate an overcritical value of the field between positively and negatively charged shells.

the star. On the contrary, $B_{\theta}$ has values exceeding the critical magnetic field near the surface of the core although localized in a narrow region between positively and negatively charged shells as expected Fig. 6. Outside the core the magnetic field becomes negative. The magnitude of the field has very small and eventually vanishing values.

In Fig. 7 the magnitude of the magnetic field is presented as a function of the rotational period $P$ on the surface of the core at the equator. Practically it demonstrates the upper limit of possible values of the magnetic field in the range between $1 \mathrm{~ms}$ and $100 \mathrm{~s}$. Fig. 8 represents magnetic lines of force inside, outside and on the surface of the star. It turned out that the lines of force of the overcritical magnetic field are appressed between two shells along the surface of the core.

In Table 1. electrodynamic properties of the globally neutral configurations with different charge distribution are presented schematically. All parameters are chosen in a such way to show the general features of the systems. Analyzing the magnetic fields of the configurations one can easily see the resemblance between the Coulomb potential $V$ and $B_{r}$, plus between the electric field $E$ and $B_{\theta}$ for both idealized and real configurations. In our case the magnetic field shares common properties with the previous configurations although it has its own peculiarities representing the smooth changes in the regions close to the surface of the core. In order to check the validity of our results we have considered similar examples. First thing when one deals with the positively and negatively charged spherical shells with the thickness of order of $\lambda_{e}$ in contrast with a radius of $10 \mathrm{~km}$ neutron star, the natural idea that comes to one's mind is to assume a spherical surface charge distribution. Since there are two spherical shells the closest example has been to explore electrodynamic properties of a spherical capacitor. As matter of fact this example showed the similar behavior of the Coulomb potential $V$ and $B_{r}$ with the solution of Thomas-Fermi equation plus a huge difference in the orders between $B_{r}$ and $B_{\theta}$, however the 
Table 1. Electrodynamic properties of the globally neutral configurations with different charge distribution are demonstrated schematically. The dashed lines correspond to the radii of the spheres.

\begin{tabular}{|c|c|c|c|}
\hline Configuration & $\begin{array}{l}\text { A spherical capacitor } \\
\text { of radii } R_{1}<R_{2}\end{array}$ & $\begin{array}{l}\text { Concentric } \\
\text { spherical layers of radii } \\
R_{1}<R_{2}<R_{3}\end{array}$ & $\begin{array}{l}\text { Our case (the solution } \\
\text { of the Thomas-Fermi } \\
\text { equation) }\end{array}$ \\
\hline $\begin{array}{l}\text { Charge } \\
\text { distribution } \rho\end{array}$ & 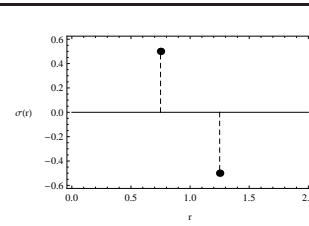 & 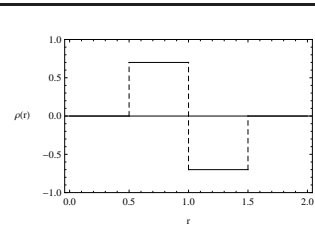 & - \\
\hline $\begin{array}{l}\text { Coulomb poten- } \\
\text { tial } V\end{array}$ & 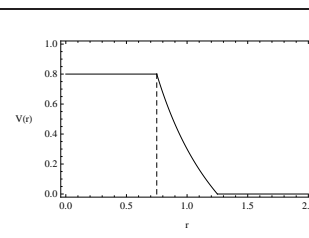 & 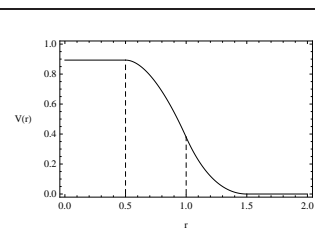 & (ve) 2.0 .4 \\
\hline Electric field $E$ & 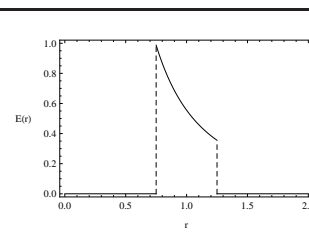 & 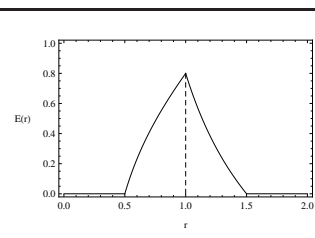 & $\begin{array}{l}1.0 \\
0.8 \\
0.0 \\
0.6 \\
0.4 \\
0.2 \\
0.0\end{array}$ \\
\hline $\begin{array}{l}\text { Radial compo- } \\
\text { nent } \\
\text { of the magnetic } \\
\text { field } B_{r}(r, 0)\end{array}$ & 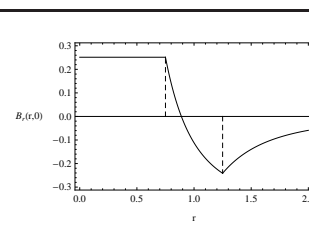 & 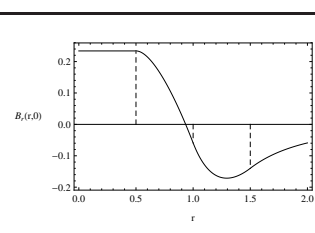 & Bats \\
\hline $\begin{array}{l}\text { Angular compo- } \\
\text { nent } \\
\text { of the magnetic } \\
\text { field } B_{\theta}\left(r, \frac{\pi}{2}\right)\end{array}$ & 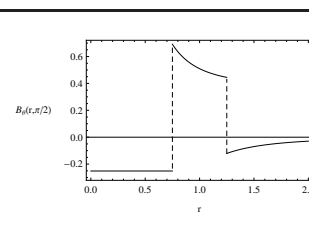 & 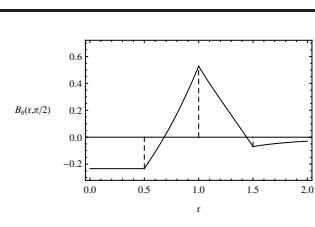 & 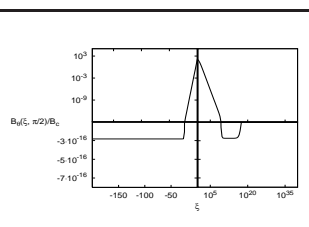 \\
\hline
\end{tabular}

electric field $E$ and $B_{\theta}$ had completely different features from our results. Moreover the spherical capacitor has not explained why in our case one had continuous $B_{\theta}$ at the boundary of the charged shells. In addition to all above given arguments there was some distance between positively and negatively charged surfaces, whereas in our case the distance between the shells was zero. Thus, one had to consider one more alternative problem close to our case in order to carry out a cross check. This time two oppositely charged spherical layers with volume charge distribution have been considered in contrast with our case. Indeed this example was fortunate and the closest simple example. It explained all the common electrodynamic properties of both systems. As a result, $B_{r}$ and $B_{\theta}$ turned out to be functions of the thickness of the spherical layers. When these thickness is of order of $\lambda_{e}$ one observes this huge 
difference and since the charge has been distributed in given volumes, with different signs, the electric field $E$ and $B_{\theta}$ turned out to be continuous.

\section{Conclusions}

In this paper we have investigated the behavior of the magnetic field induced by the rotation of a matter core of stellar dimensions on the basis of the research works considered in Ruffini et. al. ${ }^{13}$ and Popov et. al. ${ }^{18}$ using the technique developed by $\operatorname{Marsh}^{19}$.

In particular, for such a rotating system with a period of $10 \mathrm{~ms}$ we have obtained a magnetic field of order of the critical field near the surface and analyzed the magnetic lines of forces.

According to our results the magnetic field of a neutron star could be generated by its rotation as a whole rigid body leading to the formation of the constant magnetic field at the initial moments of neutron stars birth.

The problem of investigating the magnetic field in general relativity for a selfgravitating system of degenerate fermions in beta equilibrium is beyond the scope of the present work. We expect to investigate this problem in the nearest future.

\section{Acknowledgements}

The authors thank ICRANet for support and one of them (K.B.) wishes to express his deep gratitude to Jorge Rueda and Ivan Siutsou whose useful comments and critical readings promoted further improvements of the manuscript.

\section{Appendix A. Spherical Capacitor}

Consider a spherical capacitor with the internal $R_{1}$ and external $R_{2}$ radii having charges $q_{1}$ and $q_{2}$ correspondingly. The Coulomb potential in general has forms

$$
V(r)= \begin{cases}k\left(\frac{q_{1}}{R_{1}}+\frac{q_{2}}{R_{2}}\right), & r<R_{1}, \\ k\left(\frac{q_{1}}{r}+\frac{q_{2}}{R_{2}}\right), & R_{1}<r<R_{2}, \\ k\left(\frac{q_{1}}{r}+\frac{q_{2}}{r}\right), & r>R_{2}\end{cases}
$$

where

$$
k=\frac{1}{4 \pi \varepsilon_{0}} .
$$

Using the procedure developed by $\mathrm{Marsh}^{19}$ for the computation of the magnetic field one has

$$
B_{r}(r, \theta)= \begin{cases}\frac{2 k \omega \cos \theta}{3 c^{2}}\left(\frac{q_{1}}{R_{1}}+\frac{q_{2}}{R_{2}}\right), & r<R_{1}, \\ \frac{2 k \omega \cos \theta}{3 c^{2}}\left(\frac{q_{1} R_{1}^{2}}{r^{3}}+\frac{q_{2}}{R_{2}}\right), & R_{1}<r<R_{2}, \\ \frac{2 k \omega \cos \theta}{3 c^{2}}\left(\frac{q_{1} R_{1}^{2}}{r^{3}}+\frac{q_{2} R_{2}^{2}}{r^{3}}\right), & r>R_{2},\end{cases}
$$




$$
B_{\theta}(r, \theta)= \begin{cases}-\frac{2 k \omega \sin \theta}{3 c^{2}}\left(\frac{q_{1}}{R_{1}}+\frac{q_{2}}{R_{2}}\right), & r<R_{1}, \\ \frac{k \omega \sin \theta}{3 c^{2}}\left(\frac{q_{1} R_{1}^{2}}{r^{3}}-\frac{2 q_{2}}{R_{2}}\right), & R_{1}<r<R_{2}, \\ \frac{k \omega \sin \theta}{3 c^{2}}\left(\frac{q_{1} R_{1}^{2}}{r^{3}}+\frac{q_{2} R_{2}^{2}}{r^{3}}\right), & r>R_{2} .\end{cases}
$$

Let us consider a limiting case analogous to ours when

$$
q_{1}=q, \quad q_{2}=-q, \quad R_{1} \approx R_{2}-\lambda_{e} .
$$

This means that one may expand the expressions for both $B_{r}$ and $B_{\theta}$ in $\lambda_{e}$. Retaining only the terms linear in $\lambda_{e}$ one has

$$
\begin{aligned}
& B_{r}(r, \theta)= \begin{cases}\frac{2 k \omega q \lambda_{e} \cos \theta}{3 c^{2} R_{2}^{2}}, & r<R_{1}, \\
-\frac{4 k \omega q \lambda_{e} R_{2} \cos \theta}{3 c^{2} r^{3}}+\frac{2 k \omega q\left(R_{2}^{3}-r^{3}\right) \cos \theta}{3 c^{2} r^{3} R_{2}}, & R_{1}<r<R_{2}, \\
-\frac{4 k \omega q \lambda_{e} R_{2} \cos \theta}{3 c^{2} r^{3}}, & r>R_{2},\end{cases} \\
& B_{\theta}(r, \theta)= \begin{cases}-\frac{2 k \omega q \lambda_{e} \sin \theta}{3 c^{2} R_{2}^{2}}, & r<R_{1}, \\
-\frac{2 k \omega q \lambda_{e} R_{2} \sin \theta}{3 c^{2} r^{3}}+\frac{k \omega q\left(R_{2}^{3}+2 r^{3}\right) \sin \theta}{3 c^{2} r^{3} R_{2}}, & R_{1}<r<R_{2}, \\
-\frac{2 k \omega q \lambda_{e} R_{2} \sin \theta}{3 c^{2} r^{3}}, & r>R_{2} .\end{cases}
\end{aligned}
$$

Note that when $r=R_{2}$ one will have a huge difference between $B_{r}$ and $B_{\theta}$ in orders between the spherical shells.

\section{References}

1. V.L. Ginzburg, Dokl. Akad. Nauk SSSR 156, (1964) 4346 [Engl. transl.: Sov. Phys. Doklady 9, 329332].

2. L. Woltjer, ApJ. 140 (1964) 1309.

3. M. Ruderman, A\&A. 10 (1972) 427.

4. M. Ruderman, A\&SA. 16 (1995) 207-216.

5. A. Reisenegger, ApJ. 550 (2001) 860.

6. A. Reisenegger, Astron. Nachr. 328, (2007) 1173.

7. A. Reisenegger, R. Benguria, J.P. Araya, and D. Lai, A\&SA 472 (2007) 233.

8. C. Thompson and R. Duncan, ApJ 408 (1993) 194.

9. C. Thompson and R. Duncan, MNRAS 275 (1995) 255.

10. C. Thompson and R. Duncan, ApJ 473 (1996) 322.

11. R.J. Tayler, MNRAS 161 (1973) 365.

12. H. Spruit, $A \& A 381$ (2002) 923.

13. R. Ruffini, M. Rotondo and S.-S. Xue, Int. J. Mod. Phys. D 16 (2007) 1.

14. E. Lieb and B. Simon, Phys. Rev. Let. 31 (1973) 681.

15. I. Pomeranchuk and Ya. Smorodinsky, J. Phys., USSR 9 (1945) 97.

16. J. Ferreirinho, R. Ruffini and L. Stella, Phys. Lett. B 91 (1980) 314.

17. R. Ruffini and L. Stella, Phys. Lett. B 102 (1981) 442.

18. V. Popov, M. Rotondo, R. Ruffini, S. S. Xue. arxiv: 0903.3727v1.

19. J.S. Marsh, Am. J. Phys. 50 (1) (1982) 51. 